\title{
A classification of definable forcings on $\omega_{1}$
}

\author{
by
}

Jindřich Z a p let a l (Berkeley, Calif.)

\begin{abstract}
Under the assumption of the existence of sharps for reals all simply definable posets on $\omega_{1}$ are classified up to forcing equivalence.
\end{abstract}

0. Introduction. The purpose of this note is to prove

TheOREM 1. Suppose $x$ is a real, $x^{\#}$ exists and $P \in L[x]$ is a poset on $\omega_{1}$. Then $P$ is in the forcing sense equivalent to one of the following:

(1) the trivial poset,

(2) adding one Cohen real,

(3) adding $\aleph_{1}$ Cohen reals,

(4) adding a club with finite conditions [B],

(5) the collapse of $\aleph_{1}$ to $\aleph_{0}$,

(6) disjoint union of some of the above.

This theorem makes it possible to obtain full characterization of forcing properties of certain posets just from the mere syntax of their definition. Suppose sharps for reals exist - for example in the presence of a measurable cardinal - and a poset $P$ on $\omega_{1}$ has a provably $\Delta_{1}$ definition from real and ordinal parameters. Then by absoluteness $P$ is constructible from a real and the theorem can be applied. For some interesting simply definable posets see [T, JS]. In certain circumstances - like in $\sigma$-closed homogeneous forcing extensions of the model $L(\mathbb{R})$ provided this model satisfies the Axiom of Determinacy - every poset on $\omega_{1}$ definable in any way from real and ordinal parameters is constructible from a real and sharps exist. In such models then one has to use a complicated parameter to define an interesting poset of size $\aleph_{1}$.

It seems very unlikely that a similar classification theorem can be obtained for higher cardinals even though we have no definite negative result

1991 Mathematics Subject Classification: 03E40, 03E45.

Research partially supported by NSF grant no. DMS-9022140. 
in this direction. Some examples of larger simply definable posets with nontrivial properties can be found in [BJZ].

Our notation follows the set-theoretic standard as set forth in $[\mathrm{J}]$. For a poset $P$ on $\omega_{1}$ and an ordinal $\alpha \in \omega_{1}$ the expression $P \cap \alpha$ stands for the poset on $\alpha$ with the order inherited from $P$. $\dot{H}$ always denotes the canonical $P$-name for a generic filter and if $p \in P$ then by $P\lceil p$ we mean the poset $\left\{q \in P: q \leq_{P} p\right\}$. Thanks are due to the referee whose comments much simplified the original clumsy proof.

1. The proof. Suppose $x$ is a real, $x^{\#}$ exists, $P \in L[x]$ is a poset on $\omega_{1}$ and assume that $P \Vdash " \aleph_{1}$ is preserved".

LEMma 2. The following are equivalent for every $\alpha \in \omega_{1}$ and every $p \in P$ :

(1) $p \Vdash$ " $\dot{H} \cap P \cap \alpha$ meets every dense subset of $P \cap \alpha$ in $L[x]$ ",

(2) $p \Vdash$ " $\dot{H} \cap P \cap \alpha$ meets every dense subset of $P \cap \alpha$ in the ground model".

P r o of. This is in fact a consequence of a quite general fact and does not have anything to do with constructibility. Both (1) and (2) are equivalent to a first order statement about $P, P \cap \alpha$ and $p$, namely

(3) for every $q \leq p$ there is $r \in P \cap \alpha$ such that for all $s \in P \cap \alpha$ if $s \leq r$ then $s$ is compatible with $q$.

We shall show $(1) \leftrightarrow(3)$, the proof of $(2) \leftrightarrow(3)$ being similar. Suppose (3) fails. Let $q \leq p$ witness the failure and let $D=\{r \in P \cap \alpha: r$ is incompatible with $q$. The set $D \subset P \cap \alpha$ is dense, $D \in L[x]$ and $q \Vdash \check{D} \cap \dot{H}=0$. Since $q \leq p$, (1) fails. On the other hand, suppose (3) holds. Then whenever $q \leq p$ and $D \subset P \cap \alpha$ is a dense set in $L[x]$ one can find a condition $t \leq q$ with an element of $D$ above it - just choose $r \in P \cap \alpha$ witnessing (3) for $q, s \leq r$ in $D$ and the postulated lower bound $t$ of $q$ and $s$. (1) follows by a genericity argument.

Lemma 3. $P \Vdash$ "the set $\dot{D}=\left\{\alpha \in \omega_{1}: \dot{H} \cap P \cap \alpha\right.$ is a $V$-generic filter on $P \cap \alpha\}$ contains a club".

Proof. Note that by the above lemma, $P \Vdash$ " $\dot{D}=\left\{\alpha \in \omega_{1}: \dot{H} \cap P \cap \alpha\right.$ meets every maximal antichain of $P \cap \alpha$ which happens to belong to $L[x]\}$ ". Let $H \subset P$ be an arbitrary generic filter and work in $V[H]$. Let $D=\dot{D} / H$. It is enough to show that whenever $M \prec H_{\aleph_{2}}$ is a countable elementary substructure containing $x$ and $H$, then $\alpha=M \cap \omega_{1} \in D$.

Let $C$ be the closed unbounded class of $L[x]$ indiscernibles. We have $\alpha \in C$ by elementarity. Moreover, suppose that $A \subset P\lceil\alpha$ is a maximal antichain in $L[x]$. Then $A=\tau(\vec{\beta}, \alpha, \vec{\gamma})$ for some $L[x]$-Skolem term $\tau$ and 
finite sequences $\vec{\beta}, \vec{\gamma}$ of indiscernibles below, respectively above $\alpha$. Without loss of generality we can assume that $\vec{\gamma}$ are among the first $\omega$ indiscernibles in $C$ above $\omega_{1}$, and so $\vec{\gamma} \in M$. Let $B=\tau\left(\vec{\beta}, \omega_{1}, \vec{\gamma}\right)$. Thus $B \subset P$ is a maximal antichain, $B \in M$ and $A=B \cap P \cap \alpha$. By the genericity of the filter $H$ and elementarity, there is some $p \in P, p \in B \cap H \cap M$. Such a $p$ is necessarily in $P \cap \alpha$ and so in $H \cap A$; consequently, the antichain $A$ has been met. Since $A$ was arbitrary, $\alpha \in D$ and the lemma is proven.

Lemma 4. $P$ is proper.

Proof. Fix $p \in P$ and a countable elementary submodel $M \prec H_{\aleph_{2}}$ with $p, P, x \in M$. We shall produce a master condition $q \leq p$ for $M$, proving the lemma.

Let $E=\left\{\alpha \in \omega_{1}\right.$ : there is $q \leq p$ with $q \Vdash$ " $\dot{H} \cap P \cap \alpha$ is a $V$-generic filter on $P \cap \alpha$ " $\}$. Then certainly $E \subset \omega_{1}$ is stationary, since $p \Vdash$ "the closed unbounded set $\dot{D}$ from the previous lemma is included in $\check{E}$ ". Moreover, $E \in L[x]$, so $E$ must in fact contain a closed unbounded set and $M \cap \omega_{1} \in E$. Any condition $q \leq p$ witnessing the latter fact will be as required.

Conclusion 5. Suppose $P \in L[x]$ is a nowhere c.c.c. $\aleph_{1}$ preserving poset on $\omega_{1}$. Then $R O(P)$ is isomorphic to the algebra adding a club with finite conditions.

Proof. In [Z] it is proved that whenever $P$ is a nowhere c.c.c. proper poset of size $\aleph_{1}$ which forces a club of $V$-generic filters as in Lemma 3 , then $R O(P)$ is isomorphic to the algebra adding a club with finite conditions.

Conclusion 6. Suppose $P \in L[x]$ is a c.c.c. poset on $\omega_{1}$ of uniform density $\aleph_{1}$. Then $R O(P)$ is isomorphic to the algebra adding $\aleph_{1}$ Cohen reals.

Proof. From the chain condition of $P$ it follows that there is a closed unbounded set $E \subset \omega_{1}$ such that $P \Vdash$ " $\check{E} \subset \dot{D}$ ", with $\dot{D}$ being the name from Lemma 3. Thus for every $\alpha \in E$ we have $P \Vdash$ " $\dot{H} \cap P \cap \alpha$ is a $V$-generic filter", so $P \cap \alpha$ must be a regular subposet of $P$. In $[\mathrm{K}]$ it is proved that any poset of uniform density $\aleph_{1}$ with a club of regular subposets is in the forcing sense equivalent to adding $\aleph_{1}$ Cohen reals.

It is now possible to complete the proof of the theorem. Let $x$ be a real and $P \in L[x]$ be a poset on $\omega_{1}$. Choose a maximal antichain $A \subset P$ so that each $p \in A$ has exactly one of the following properties:

(1) all conditions below $p$ are pairwise compatible,

(2) $P\lceil p$ has countable density and $p$ is not compatible with any condition as in (1),

(3) $P\lceil p$ has c.c.c. and $p$ is not compatible with any condition as in (1) or $(2)$, 
(4) $p \Vdash$ " $\aleph_{1}$ is preserved" and $p$ is not compatible with any condition as in $(1),(2)$ or $(3)$,

(5) $p \Vdash$ " $\aleph_{1}$ is collapsed".

So for $p \in A$ either (1) holds and then $R O(P\lceil p)$ is trivial; or (2) holds and then $R O(P\lceil p)$ is isomorphic to adding one Cohen real; or (3) holds and then $R O\left(P\lceil p)\right.$ is isomorphic to adding $\aleph_{1}$ Cohen reals by Conclusion 6; or (4) holds and $R O(P\lceil p)$ is isomorphic to adding a club with finite conditions by Conclusion 5; or (5) holds and then $R O(P \backslash p)$ is isomorphic to the collapse of $\aleph_{1}$ by [J, Lemma 25.11]. The theorem follows.

\section{References}

[B] J. Baumgartner, Applications of the proper forcing axiom, in: Handbook of Set-Theoretic Topology, K. Kunen and J. E. Vaughan (eds.), North-Holland, Amsterdam, 1984, 913-959.

[BJZ] B. Balcar, T. Jech and J. Zapletal, Generalizations of Cohen algebras, Ann. Pure Appl. Logic, to appear.

[J] T. Jech, Set Theory, Academic Press New York, 1978.

[JS] T. Jech and S. Shelah, Possible PCF algebras, J. Symbolic Logic 61 (1996), 313-318.

[K] S. Koppelberg, Characterization of Cohen algebras, in: Papers on General Topology and Applications, S. Andima, R. Kopperman, P. R. Misra and A. R. Todd (eds.), Ann. New York Acad. Sci. 704, 1993, 227-237.

[S] S. Shelah, Proper Forcing, Springer, New York, 1982.

[T] S. Tennenbaum, Souslin's problem, Proc. Nat. Acad. Sci. U.S.A. 59 (1968), $60-63$.

[Z] J. Zapletal, Characterization of the club forcing, in: Papers on General Topology and Applications, S. Andima, R. C. Flagg, G. Itzkowitz, Y. Kong, R. Kopperman and P. Misra (eds.), Ann. New York Acad. Sci. 806, 1996, 476-484.

M.S.R.I.

1000 Centennial Drive

Berkeley, California 94720

U.S.A.

E-mail: jindra@msri.org 